\title{
AN ALGEBRAIC MULTIPLICITY THEORY FOR ANALYTIC OPERATOR-VALUED FUNCTIONS
}

\author{
JÓN ARASON and ROBERT MAGNUS
}

\section{Contents.}

1. Introduction

2. Examination of the quotient

3. Multiplicity axioms

4. Suspension equivalence

5. Some examples

6. The canonical linearization

7. Homotopy invariance

8. Estimates of multiplicity

\section{Introduction.}

An analytic operator-valued function $A$ is an analytic mapping $A: D \rightarrow L(E, E)$, where the domain $D=D(A)$ of $A$ is an open subset of the complex plane $\mathrm{C}$, and where $E=E(A)$ is a complex Banach space. For such a function $A$ we denote by $\Sigma(A)$ the singular set of $A$; that is, the set of points $z \in D$ such that $A(z)$ is not invertible. It is a relatively closed subset of $D$.

A multiplicity theory $m$ for analytic operator-valued functions assigns to every analytic operator-valued function $A$ and to every isolated compact subset $C$ of $\Sigma(A)$ an element $m_{C}(A)$ of a fixed additive semi-group $M$ such that certain natural conditions (or axioms) are satisfied. We then refer to $m_{C}(A)$ as the multiplicity of $A$ at $C$. 
In a previous paper [3] such a multiplicity theory was constructed taking its values in the semigroup of isomorphism classes of complex Banach spaces. In this article we describe a new way to define multiplicity with values in the same semigroup. We believe that it recommends itself by its naturalness. It turns out that this gives the same multiplicity as in [3], although the approach is completely different. The proofs of the relevant properties are independent of the previous paper.

In our exposition we shall initially take a point of view which differs slightly from that outlined above. Instead of an isolated compact subset $C$ of $\Sigma(A)$ we shall work with a bounded open subset $\Omega$ of $\mathrm{C}$ such that $\bar{\Omega} \subset D$ and $\partial \Omega \cap \Sigma(A)=\emptyset$. We then say that $\Omega$ is admissible for $A$ and refer to $(A, \Omega)$ as an admissible pair. We shall write $\Sigma_{\Omega}(A)=\Omega \cap \Sigma(A)$. Then $\Sigma_{\Omega}(A)$ is an isolated compact subset of $\Sigma(A)$ and every isolated compact subset of $\Sigma(A)$ arises in this way.

A multiplicity theory $m$ with values in $M$ now assigns to each admissible pair $(A, \Omega)$ an element $m(A, \Omega)$ of $M$. The axioms which we consider it reasonable to impose on $m$ will be discussed in section 3 .

We define our multiplicity theory in the following way. Let $A: D \rightarrow L(E, E)$ be analytic and let $\Omega$ be admissible for $A$. Let $\mathscr{H}(\Omega, E)$ denote the space of functions from $\bar{\Omega}$ to $E$ which are analytic in $\Omega$ and continuous on $\bar{\Omega}$. Equipped with the supremum norm this becomes a Banach space. Let $A \mathscr{H}(\Omega, E)$ denote the set of all elements of $\mathscr{H}(\Omega, E)$ of the form Af for some $f \in \mathscr{H}(\Omega, E)$. We define $m(A, \Omega)$ to be the isomorphism class of the Banach space $\mathscr{H}(\Omega, E) / A \mathscr{H}(\Omega, E)$. That the quotient is a Banach space will become clear in the next section. We shall often denote the quotient space by $\mathscr{H}_{A}(\Omega, E)$.

We shall prove that this is indeed a multiplicity theory. We shall also see that - identifying the isomorphism class of a finite-dimensional space with its dimension - this gives the usual multiplicity for $\mathrm{C}$-valued functions, that is, the sum of the multiplicities of the zeros of $A$ in $\Omega$. If $A$ takes its values in the set of Fredholm operators of index 0 , then $m(A, \Omega)$ is the sum of the multiplicities of the singular points of $A$ in $\Omega$. These multiplicities have been defined in various, equivalent, ways in the literature; see [4], [5], [6] - this is just a sample - the concept seems to be considerably older.

We shall show that our present theory gives the same multiplicity as that defined in [3]. There the construction depended on the concept of suspensionequivalence, or s-equivalence for short. This notion will be explained in due course. It is an equivalence relation for admissible pairs. In [3] it was necessary to know that any admissible pair $(A, \Omega)$ could be reduced by s-equivalence to an admissible pair $(B, \Omega)$, where $B(z)=z I_{F}-T, F=E(B)$, and $T \in L(F, F)$. Such a reduction is called a linearization. We shall describe a 
linearization, which is strikingly natural and elegant, which we call the canonical linearization.

We shall show that $m(A, \Omega)$ is not very large compared to $E=E(A)$; more precisely it is the isomorphism class of a complemented subspace of a finite power of $E$.

In a succeeding paper we intend to study multiplicity theories with more general semi-groups; and, among other things, we shall define a universal multiplicity theory from which all others can be derived.

After this paper was written it was brought to our notice that the fact that the canonical linearization is a linearization can be derived rather easily from results of Kaashoek, van der Mee and Rodman [2]. While giving due credit to those authors we feel it an advantage to present short and virtually self-contained proofs of our results. Our concept of a multiplicity theory is new and we consider that the algebraic approach introduces an interesting and fruitful method into the study of analytic operator-valued functions.

\section{Examination of the quotient.}

In this section we work with a fixed admissible pair $(A, \Omega)$. We write $D=D(A)$ and $E=E(A)$.

In addition to $\mathscr{H}(\Omega, E)$ there are several other spaces of analytic functions associated with $\Omega$ which can be used to form a quotient. For any subset $U$ of $\overline{\mathrm{C}}=\mathrm{C} \cup\{\infty\}$ let $\mathcal{O}(U, E)$ denote the space of analytic mappings defined on an open neighbourhood of $U$ with values in $E$. We identify two mappings which agree on a neighbourhood of $U$. Thus $\mathcal{O}(U, E)$ consists of germs localized at $U$. If $U$ is open then $\mathcal{O}(U, E)$ consists simply of all analytic $E$-valued mappings with domain $U$.

We have the natural embeddings induced by restriction

$$
\mathcal{O}(\bar{\Omega}, E) \longrightarrow \mathscr{H}(\Omega, E) \longrightarrow \mathcal{O}(\Omega, E)
$$

but these mappings are not vector-space isomorphisms. They map $A \mathscr{O}(\bar{\Omega}, E)$ to $A \mathscr{H}(\Omega, E)$, and $A \mathscr{H}(\Omega, E)$ to $A \mathcal{O}(\Omega, E)$ respectively. Hence they induce linear maps between the quotient spaces

$$
\frac{\mathcal{O}(\bar{\Omega}, E)}{A \mathcal{O}(\bar{\Omega}, E)} \longrightarrow \frac{\mathscr{H}(\Omega, E)}{A \mathscr{H}(\Omega, E)} \longrightarrow \frac{\mathcal{O}(\Omega, E)}{A \mathcal{O}(\Omega, E)}
$$

It will transpire that these are vector-space isomorphisms. Since, as will be shown, the second space carries a Banach-space structure, we may regard the other quotients as Banach-spaces also.

For each $z$ in $D$ let $A_{z}$ be the unique analytic function which satisfies 


$$
A(w)=A(z)+(w-z) A_{z}(w)
$$

for all $w \in D$. Then $A_{z}(w)=(A(w)-A(z)) /(w-z)$ if $w \neq z$. Clearly the mapping $(w, z) \mapsto A_{z}(w)$ is analytic on $D \times D$. Moreover by Cauchy's formula we have

$$
A_{z}(w)=\frac{1}{2 \pi i} \int_{\partial \Omega^{\prime \prime}} \frac{A(\sigma)}{(\sigma-z)(\sigma-w)} d \sigma
$$

where $\Omega^{\prime \prime}$ is here any Cauchy-domain within $D$ which contains $z$ and $w$.

Let $f \in \mathcal{O}(\Omega, E)$. For each $z \in D$ we define $g_{1}(z) \in E$ asfollows. Choose a Cauchy-domain $\Omega^{\prime}$ containing $\Sigma_{\Omega}(A)$ but such that $\overline{\Omega^{\prime}} \subset \Omega$. Then set

$$
g_{1}(z)=\frac{1}{2 \pi i} \int_{\partial \Omega^{\prime}} A_{z}(\zeta) A(\zeta)^{-1} f(\zeta) d \zeta
$$

It clear that $g_{1}$ is an analytic function on $D$ and that it does not depend on the choice of $\Omega^{\prime}$ subject to the stated conditions. That a Cauchy-domain can be found to satisfy these conditions is quite well known. A proof can be found in [7].

Let $f \in \mathcal{O}(\Omega, E)$. For each $z \in \Omega$ we define $g_{2}(z) \in E$ as follows. Choose a Cauchy-domain $\Omega^{\prime}$ containing $\Sigma_{\Omega}(A)$ but such that $\overline{\Omega^{\prime}} \subset \Omega$ and $z \in \Omega^{\prime}$. Then set

$$
g_{2}(z)=\frac{1}{2 \pi i} \int_{\partial \Omega^{\prime}} \frac{A(\zeta)^{-1} f(\zeta)}{\zeta-z} d \zeta
$$

It is clear that $g_{2}(z)$ does not depend on the choice of $\Omega^{\prime}$ subject to the stated conditions. It then follows that $g_{2}$ is analytic on $\Omega$.

Let $f \in \mathcal{O}(\Omega, E)$. For each $z \in \mathrm{C} \backslash \Sigma_{\Omega}(A)$ we define $h(z) \in E$ as follows. Choose a Cauchy-domain $\Omega^{\prime}$ containing $\Sigma_{\Omega}(A)$ but such that $\overline{\Omega^{\prime}} \subset \Omega$ and $z \notin \overline{\Omega^{\prime}}$. Then set

$$
h(z)=\frac{1}{2 \pi i} \int_{\partial \Omega^{\prime}} \frac{A(\zeta)^{-1} f(\zeta)}{z-\zeta} d \zeta
$$

It is clear that $h(z)$ is independent of the choice of $\Omega^{\prime}$ subject to the stated conditions. It then follows that $h$ is analytic on $\left(\overline{\mathbf{C}} \backslash \Sigma_{\Omega}(A)\right)$ with $h(\infty)=0$.

When we want to make the dependence of $g_{1}, g_{2}$ and $h$ on $f$ explicit, we write $g_{1}=G_{1} f, \quad g_{2}=G_{2} f$ and $h=H f$. Then $G_{1}: \mathcal{O}(\Omega, E) \rightarrow \mathcal{O}(D, E)$, $G_{2}: \mathcal{O}(\Omega, E) \rightarrow \mathcal{O}(\Omega, E)$ and $H: \mathcal{O}(\Omega, E) \rightarrow \mathcal{O}\left(\overline{\mathbf{C}} \backslash \Sigma_{\Omega}(A), E\right)$ are linear maps.

Lemma 1. (i) For each $z \in \Omega$ we have

$$
f(z)=g_{1}(z)+A(z) g_{2}(z)
$$


(ii) For each $z \in \Omega \backslash \Sigma_{\Omega}(A)$ we have

$$
g_{1}(z)=A(z) h(z)
$$

Proof. Let $z \in D$. Choose $\Omega^{\prime}$ in the definition of $g_{1}(z)$ such that $z \notin \partial \Omega^{\prime}$. Then we have

$$
\begin{aligned}
g_{1}(z) & =\frac{1}{2 \pi i} \int_{\partial \Omega^{\prime}} \frac{A(\zeta)-A(z)}{\zeta-z} A(\zeta)^{-1} f(\zeta) d \zeta \\
& =\frac{1}{2 \pi i} \int_{\partial \Omega^{\prime}} \frac{f(\zeta)}{\zeta-z} d \zeta-A(z) \frac{1}{2 \pi i} \int_{\partial \Omega^{\prime}} \frac{A(\zeta)^{-1} f(\zeta)}{\zeta-z} d \zeta
\end{aligned}
$$

Let $z \in \Omega$. Then we can choose $\Omega^{\prime}$ so that $z \in \Omega^{\prime}$. We conclude, by Cauchy's formula, that $g_{1}(z)=f(z)-A(z) g_{2}(z)$.

Next let $z \notin \Sigma_{\Omega}(A)$. Now we choose $\Omega^{\prime}$ so that $z \notin \overline{\Omega^{\prime}}$. This time Cauchy's formula gives $g_{1}(z)=A(z) h(z)$.

From lemma 1 (i) and the fact that $A$ is invertible in a neighbourhood of $\partial \Omega$, we see that $f \in \mathscr{H}(\Omega, E)$ implies $g_{2} \in \mathscr{H}(\Omega, E)$, and $f \in \mathcal{O}(\bar{\Omega}, E)$ implies $g_{2} \in \mathcal{O}(\bar{\Omega}, E)$. As $g_{1}$ always lies in $\mathcal{O}(D, E)$, we conclude that every element in each of the quotient spaces $\mathcal{O}(\bar{\Omega}, E) / A \mathcal{O}(\bar{\Omega}, E), \mathscr{H}(\Omega, E) / A \mathscr{H}(\Omega, E)$ and $\mathcal{O}(\Omega, E) / A \mathcal{O}(\Omega, E)$, is represented by an element of $\mathcal{O}(D, E)$. Hence the linear maps

$$
\frac{\mathcal{O}(\bar{\Omega}, E)}{A \mathcal{O}(\bar{\Omega}, E)} \longrightarrow \frac{\mathscr{H}(\Omega, E)}{A \mathscr{H}(\Omega, E)} \longrightarrow \frac{\mathcal{O}(\Omega, E)}{A \mathcal{O}(\Omega, E)}
$$

induced by restriction, are surjective. But using the fact that $A$ is invertible in a neighbourhood of $\partial \Omega$ we see that these maps are also injective. The following is therefore proved.

THEOREM 1. The induced mappings in the diagram

$$
\frac{\mathcal{O}(\bar{\Omega}, E)}{A \mathcal{O}(\bar{\Omega}, E)} \longrightarrow \frac{\mathscr{H}(\Omega, E)}{A \mathscr{H}(\Omega, E)} \longrightarrow \frac{\mathcal{O}(\Omega, E)}{A \mathcal{O}(\Omega, E)}
$$

are vector-space isomorphisms.

For each $f \in \mathcal{O}(\Omega, E)$ we can define an element $S f$ of $\mathcal{O}(\Omega, E)$ by restricting $g_{1}$ to $\Omega$. This gives a linear map $S: \mathcal{O}(\Omega, E) \rightarrow \mathcal{O}(\Omega, E)$. In the same way we get linear maps from $\mathscr{H}(\Omega, E)$ to itself, and from $\mathcal{O}(\bar{\Omega}, E)$ to itself. We shall denote all these maps by $S$, leaving the context to make clear which spaces are involved. 
Lemma 2. Let $X$ stand for any of the spaces $\mathcal{O}(\Omega, E)$, $\mathscr{H}(\Omega, E)$ or $\mathcal{O}(\bar{\Omega}, E)$. The map $S: X \rightarrow X$ is a projection and $\operatorname{ker} S=A X$.

Proof. We first observe that if $f \in A X$, then $g_{1}=0$ by Cauchy's theorem. Applying $S$ to the identity $f=g_{1}+A g_{2}=S f+A g_{2}$ now gives $S f=S^{2} f$. Hence $S$ is a projection. Moreover $S f=0$ if and only if $f=A g_{2}$.

We recall that $\mathscr{H}(\Omega, E)$ is a Banach space.

Lemma3. The projection $S: \mathscr{H}(\Omega, E) \rightarrow \mathscr{H}(\Omega, E)$ is continuous.

Proof In the formula (1) for $g_{1}$ we fix the domain $\Omega^{\prime}$ independently of $f \in \mathscr{H}(\Omega, E)$. Then we get, since $A_{z}(\zeta)$ is bounded for $(z, \zeta) \in \bar{\Omega} \times \partial \Omega^{\prime}$,

$$
\left\|g_{1}(z)\right\|<C \sup _{\zeta \in \partial \Omega^{\prime}}\|f(\zeta)\|
$$

for all $z \in \bar{\Omega}$, where $C$ is independent of $f$ and $z$. In particular we deduce

$$
\|S f\|<C|| f \|
$$

Hence $S$ is continuous.

We conclude that the range of $S: \mathscr{H}(\Omega, E) \rightarrow \mathscr{H}(\Omega, E)$ is a closed subspace of $\mathscr{H}(\Omega, E)$ and that the quotient $\mathscr{H}(\Omega, E) / \operatorname{ker} S=\mathscr{H}(\Omega, E) /$ $A \mathscr{H}(\Omega, E)=\mathscr{H}_{A}(\Omega, E)$ is a Banach space isomorphic to ran $S$. Hence the other quotient spaces in theorem 1 can be viewed as Banach spaces also.

Next we set forth an interesting characterization of the range of $S$. To be explicit, we will consider $S: \mathcal{O}(\Omega, E) \rightarrow \mathcal{O}(\Omega, E)$. The kernel of $S$ can be viewed as the set of all $f \in \mathcal{O}(\Omega, E)$ with the property that the mapping $\Omega \backslash \Sigma_{\Omega}(A) \ni z \mapsto A(z)^{-1} f(z)$ has an analytic extension to $\Omega$. There exists a similar characterization of the range of $S$.

THEOREM 2. The range of the projection $S: \mathcal{O}(\Omega, E) \rightarrow \mathcal{O}(\Omega, E)$ consists of all $f \in \mathcal{O}(\Omega, E)$ with the property that the mapping $\Omega \backslash \Sigma_{\Omega}(A) \ni z \mapsto$ $A(z)^{-1} f(z)$ has an analytic extensionto $\overline{\mathrm{C}} \backslash \Sigma_{\Omega}(A)$ which vanishes at infinity.

Proof. Let $f \in \mathcal{O}(\Omega, E)$. By lemma 1 (ii) we have $S f=g_{1}=A h$ where $h$ is analytic on $\overline{\mathrm{C}} \backslash \Sigma_{\Omega}(A)$ and $h(\infty)=0$.

Conversely suppose that $f$ has the property in question. Let $k$ be the analytic extension of the mapping $\Omega \backslash \Sigma_{\Omega}(A) \ni z \mapsto A(z)^{-1} f(z)$ to $\overline{\mathrm{C}} \backslash \Sigma_{\Omega}(A)$ such that $k(\infty)=0$. Formula (2) (where $z \in \Omega^{\prime}$ ) gives

$$
g_{2}(z)=\frac{1}{2 \pi i} \int_{\partial \Omega^{\prime}} \frac{A(\zeta)^{-1} f(\zeta)}{\zeta-z} d \zeta=\frac{1}{2 \pi i} \int_{\partial \Omega^{\prime}} \frac{k(\zeta)}{\zeta-z} d \zeta
$$

Now we may replace the contour $\partial \Omega^{\prime}$ by a large circle whose radius tends to 
infinity. We conclude that $g_{2}(z)=0$ for each $z \in \Omega$. But then $f$ is in the range of $S$.

Theorem 3. $m(A, \Omega)$ depends only on $A$ and $\Sigma_{\Omega}(A)$.

Proof. It suffices to show that if $\Omega_{1}$ and $\Omega_{2}$ are both admissible for $A$, and $\Sigma_{\Omega_{1}}(A)=\Sigma_{\Omega_{2}}(A)$, then $\mathscr{H}_{A}\left(\Omega_{1}, E\right)$ is isomorphic to $\mathscr{H}_{A}\left(\Omega_{2}, E\right)$.

Consider first the case when $\Omega_{2} \subset \Omega_{1}$. Then just as in the proof of theorem 1 the restriction map $\mathscr{H}\left(\Omega_{1}, E\right) \rightarrow \mathscr{H}\left(\Omega_{2}, E\right)$ induces a vector-space isomorphism $\alpha: \mathscr{H}_{A}\left(\Omega_{1}, E\right) \rightarrow \mathscr{H}_{A}\left(\Omega_{2}, E\right)$. However the restriction map is also continuous. Hence $\alpha$ is a Banach-space isomorphism.

For the general case we apply the result of the last paragraph to conclude that $\mathscr{H}_{A}\left(\Omega_{1}, E\right)$ and $\mathscr{H}_{A}\left(\Omega_{2}, E\right)$ are both isomorphic as Banach spaces to $\mathscr{H}_{A}\left(\Omega_{1} \cap \Omega_{2}, E\right)$.

Consider the space $\mathcal{O}\left(\Sigma_{\Omega}(A), E\right)$. Because $\Omega$ may have components containing no singular points the restriction map from $\mathcal{O}(\Omega, E)$ to $\mathcal{O}\left(\Sigma_{\Omega}(A), E\right)$ need not be injective. However the following holds, and its proof is the same as that of theorem 1 .

Lemma 4. The map

$$
\frac{\mathcal{O}(\Omega, E)}{A \mathcal{O}(\Omega, E)} \longrightarrow \frac{\mathcal{O}\left(\Sigma_{\Omega}(A), E\right)}{A \mathcal{O}\left(\Sigma_{\Omega}(A), E\right)}
$$

induced by restriction is an isomorphism of vector spaces.

Since $\Omega$ is irrelevant, perhaps the nicest way to think of the multiplicity is as the quotient space $\mathcal{O}(C, E) / A \mathcal{O}(C, E)$ where $C=\Sigma_{\Omega}(A)$. However there is a Banach-space structure which is not apparent in this formulation. This structure can be realized by any one of a set of equivalent norms each determined by choosing a set $\Omega$ which is admissible for $A$ and satisfies $\Omega \cap \Sigma(A)=C$. For explicit calculations we can take the norm of the element $f+A \mathcal{O}(C, E)$ to be $\sup _{z \in \bar{\Omega}}\left\|g_{1}(z)\right\|$, where $g_{1}$ is defined by formula (1), taking $\Omega^{\prime}$ to lie within the domain of $f$.

\section{Multiplicity axioms.}

We propose the following as reasonable axioms for a multiplicity theory.

1. If $T: E \rightarrow F$ is an isomorphism of Banach spaces then

$$
m\left(T A T^{-1}, \Omega\right)=m(A, \Omega)
$$

2. If $\Sigma_{\Omega}(A)=\emptyset$ then $m(A, \Omega)=0$. 
3. If $E(A)=E(B)$ and $\Omega$ is admissible for $A$ and $B$ then

$$
m(A B, \Omega)=m(A, \Omega)+m(B, \Omega)
$$

4. If $\Omega$ is admissible for $A$ and $B$ then

$$
m(A \oplus B, \Omega)=m(A, \Omega)+m(B, \Omega)
$$

5. If $\Omega_{1}$ and $\Omega_{2}$ are admissible for $A$ and $\Omega_{1} \cap \Omega_{2}=\emptyset$ then

$$
m\left(A, \Omega_{1} \cup \Omega_{2}\right)=m\left(A, \Omega_{1}\right)+m\left(A, \Omega_{2}\right)
$$

6. $m(A, \Omega)$ depends only on $A \mid \Omega$ and $\Omega$.

7. $m(A, \Omega)$ depends only on $A$ and $\Sigma_{\Omega}(A)$.

Axiom 3 is called the product theorem, while axiom 5 is called additivity on sets.

Certain properties additional to those listed as axioms are often desirable. Axiom 2 can be strengthened by requiring that $m(A, \Omega)=0$ if and only if $\Sigma_{\Omega}(A)=\emptyset$. Another is homotopy invariance: if we deform $A$ continuously in such a way that $\Omega$ is always admissible, then $m(A, \Omega)$ remains unchanged. We shall establish these properties for our theory, but do not regard them as axioms.

We begin by carrying out the easier verifications.

Theorem 4. The multiplicity theory $m$ satisfies axioms 1, 2, 4, 6 and 7.

Proof. Axiom 1: Let $T: E \rightarrow F$ be an isomorphism. Then the map $\mathscr{H}(\Omega, E) \ni f \mapsto T f$ is an isomorphism of $\mathscr{H}(\Omega, E)$ onto $\mathscr{H}(\Omega, F)$ which induces an isomorphism of $\mathscr{H}_{A}$ onto $\mathscr{H}_{T A T^{-1}}$.

Axiom 2: If $\Sigma_{\Omega}(A)=\emptyset$ then $A$ is invertible on $\bar{\Omega}$ and $A \mathscr{H}(\Omega, E)=$ $\mathscr{H}(\Omega, E)$.

Axiom 6: Obvious from the definition.

Axiom 7: Follows from theorem 3.

Axiom 4: Write $E=E(A)$ and $F=E(B)$. Then $E(A \oplus B)=E \oplus F$. In obvious ways we can assert that $\mathscr{H}(\Omega, E \oplus F)=\mathscr{H}(\Omega, E) \oplus \mathscr{H}(\Omega, F)$ and $(A \oplus B) \mathscr{H}(\Omega, E \oplus F)=A \mathscr{H}(\Omega, E) \oplus B \mathscr{H}(\Omega, F)$. Hence $\mathscr{H}_{A \oplus B}(\Omega, E \oplus F)=$ $\mathscr{H}_{A}(\Omega, E) \oplus \mathscr{H}_{B}(\Omega, F)$.

In fact the theory $m$ satisfies axiom 2 in the strengthened form: $m(A, \Omega)=0$ if and only if $\Sigma_{\Omega}(A)=\emptyset$. Although it is not hard to see this directly, the neatest way is to use the canonical linearization which is discussed in a later section.

THEOREM 5 (Additivity on sets). The multiplicity theory $m$ satisfies axiom 5.

Proof. Let $\Omega_{1}$ and $\Omega_{2}$ be admissible for $A$ and assume that $\Omega_{1} \cap \Omega_{2}=\emptyset$. 
Let $\Omega=\Omega_{1} \cup \Omega_{2}$. We must show that $m(A, \Omega)=m\left(A, \Omega_{1}\right)+m\left(A, \Omega_{2}\right)$. We know that $m(A, \Omega)$ only depends on $\Sigma_{\Omega}(A)$ and not on $\Omega$ as such. Therefore we may shrink $\Omega_{1}$ and $\Omega_{2}$ and so, without loss of generality, we can assume that $\bar{\Omega}_{1} \cap \bar{\Omega}_{2}=\emptyset$. Now we have a Banach-space isomorphism $\mathscr{H}(\Omega, E) \rightarrow \mathscr{H}\left(\Omega_{1}, E\right) \oplus \mathscr{H}\left(\Omega_{2}, E\right)$ given by $f \mapsto\left(f\left|\bar{\Omega}_{1}, f\right| \bar{\Omega}_{2}\right)$. This maps $A \mathscr{H}(\Omega, E)$ to $A \mathscr{H}\left(\Omega_{1}, E\right) \oplus A \mathscr{H}\left(\Omega_{2}, E\right)$, and hence induces a continuous surjective map $\mathscr{H}_{A}(\Omega, E) \rightarrow \mathscr{H}_{A}\left(\Omega_{1}, E\right) \oplus \mathscr{H}_{A}\left(\Omega_{2}, E\right)$ which is plainly also injective.

Finally we prove the product theorem-axiom 3 .

Let $A: D \rightarrow L(E, E)$ and $B: D \rightarrow L(E, E)$ be analytic with $\Omega$ admissible for both $A$ and $B$. Abbreviating $\mathscr{H}(\Omega, E)$ to $\mathscr{H}$, we have the linear mappings

$$
\mathscr{H} \stackrel{\kappa}{\longrightarrow} \mathscr{H} \stackrel{\tau}{\longrightarrow} \mathscr{H}
$$

where $\kappa$ is multiplication by $A$ and $\tau$ is the identity. Note that $\kappa$ is injective, since $A f=0$ implies that $f(z)=0$ in a neighbourhood of the boundary $\partial \Omega$, so that $f=0$ throughout $\Omega$. The mappings $\kappa$ and $\tau$ induce mappings

$$
0 \longrightarrow \frac{\mathscr{H}}{B \mathscr{H}} \stackrel{\bar{\kappa}}{\longrightarrow} \frac{\mathscr{H}}{A B \mathscr{H}} \stackrel{\bar{\tau}}{\longrightarrow} \frac{\mathscr{H}}{A \mathscr{H}} \longrightarrow 0
$$

Clearly $\bar{\tau} \bar{\kappa}=0, \bar{\tau}$ is surjective and $\bar{\kappa}$ is injective. Moreover the range of $\bar{\kappa}$ is the kernel of $\bar{\tau}$. For if $f+A \mathscr{H}=0$ then $f=A h$ for some $h$; then $f+A B \mathscr{H}=A h+A B \mathscr{H}=\bar{\kappa}(h+B \mathscr{H})$. The sequence (4) is therefore exact. We shall now show that $\bar{\tau}$ has a continuous right-inverse. Let $\pi_{A}$ [resp. $\pi_{A B}$ ] denote the canonical mapping from $\mathscr{H}$ to $\mathscr{H} / A \mathscr{H}$ [resp. $\mathscr{H} / A B \mathscr{H}]$. As shown in section 2 , there is a linear map $l$, given by $l(f+A \mathscr{H})=S f$, such that the diagram

$$
\begin{array}{rll}
\frac{\mathscr{H}}{A \mathscr{H}} & \stackrel{l}{\longrightarrow} & \stackrel{\mathscr{H}}{ } \\
I & \searrow & \mid \pi_{A} \\
& & \frac{\mathscr{H}}{A \mathscr{H}}
\end{array}
$$

commutes, while the diagram

$$
\begin{array}{ccc}
\mathscr{H} & \stackrel{\pi_{A B}}{\longrightarrow} & \frac{\mathscr{H}}{A B \mathscr{H}} \\
\pi_{A} \downarrow & \swarrow \bar{\tau} & \\
\frac{\mathscr{H}}{A \mathscr{H}} & &
\end{array}
$$

plainly commutes. Combining the two we see that $\pi_{A B} l$ is a right-inverse to 
$\bar{\tau}$. The exact sequence (4) therefore splits and we deduce that $\mathscr{H} / A B \mathscr{H}$ is isomorphic to the direct sum of $\mathscr{H} / A \mathscr{H}$ and $\mathscr{H} / B \mathscr{H}$. This is the product theorem. We state this formally.

Theorem 6 (Product theorem). If $\Omega$ is admissible for both $A$ and $B$ then $m(A B, \Omega)=m(A, \Omega)+m(B, \Omega)$.

\section{Suspension equivalence.}

In the paper [3] a central role was played by the concepts of equivalence and suspension-equivalence (abbreviated to s-equivalence).

Let $E$ and $F$ be Banach spaces, $A: D \rightarrow L(E, E)$ an analytic mapping, and $T \in L(F, E)$ an isomorphism. By axiom 1 we have $m(A, \Omega)=m\left(T^{-1} A T, \Omega\right)$. Combining this with a special case of the product theorem we obtain the following result.

Theorem 7. Let $\phi: D \rightarrow L(E, F)$ and $\psi: D \rightarrow L(E, F)$ be analytic mappings which take invertible values only. If $B: D \rightarrow L(F, F)$ and $A: D \rightarrow L(E, E)$ satisfy $\phi A=B \psi$, and $\Omega$ is admissible for both $A$ and $B$, then $m(A, \Omega)=m(B, \Omega)$.

In fact the isomorphism from $\mathscr{H}_{A}(\Omega, E)$ to $\mathscr{H}_{B}(\Omega, F)$ is induced by the linear map $f \mapsto \phi f$ from $\mathscr{H}(\Omega, E)$ to $\mathscr{H}(\Omega, F)$.

Analytic operator-valued mappings $A$ and $B$ which satisfy the conditions of theorem 7 are said to be equivalent. We see that invariance of multiplicity under equivalence is a consequence of axiom 1 and the product theorem (axiom 3).

Let $X$ be a Banach space. The operator function $A \oplus I_{X}$ is called the $X$ suspension of $A$ by analogy with its namesake in topology, (elsewhere in the literature it is called the $X$-extension of $A$ ). If we do not wish to mention $X$ explicitly we refer simply to a suspension of $A$.

Suspension-equivalence combines the simpler concepts of suspension and equivalence. Analytic operator functions $A$ and $B$ are s-equivalent if there exist Banach spaces $X$ and $Y$ such that $A \oplus I_{X}$ and $B \oplus I_{Y}$ are equivalent. More explicitly, there should exist operator-valued analytic mappings $\phi: D \rightarrow L(E \oplus X, F \oplus Y)$ and $\psi: D \rightarrow L(E \oplus X, F \oplus Y)$, both of which take invertible values only, such that

$$
\phi\left(A \oplus I_{X}\right)=\left(B \oplus I_{Y}\right) \psi
$$

It follows at once from the axioms and theorem 7 (itself a consequence of the axioms), that $m(A, \Omega)=m(B, \Omega)$ if $A$ and $B$ are s-equivalent, a result which holds for all multiplicity theories. We proceed to generalize the conclusion of the preceding paragraph for our theory $m$. Let $A: D \rightarrow L(E, E)$ and 
$B: D \rightarrow L(F, F)$ be analytic mappings. Suppose that we have a commutative diagram

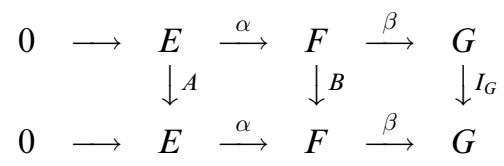

with exact rows. Then we say that $B$ is a weak suspension of $A$. In the diagram, a morphism (arrow) means an analytic mapping from $D$ to the space of bounded linear mappings from the initial space to the final space. Exactness of the rows means that for each $z \in D$ the sequence of linear maps

$$
0 \longrightarrow E \stackrel{\alpha(z)}{\longrightarrow} F \stackrel{\beta(z)}{\longrightarrow} G
$$

is exact.

The concept of weak suspension, which was introduced in [3], with, though, the additional requirement that $\beta(z)$ was surjective, generalizes the concept of suspension. We recall that $B$ is a suspension of $A$ if $B=A \oplus I_{G}$ for some $G$. This is a weak suspension where $F=E \oplus G, \alpha(z)$ is the injection $E \rightarrow E \oplus G$ mapping $u$ to $u \oplus 0_{G}$, and $\beta(z)$ is the surjection $E \oplus G \rightarrow G$ mapping $u \oplus v$ to $v$.

THEOREM 8. Let $B$ be a weak suspension of $A$ and let $\Omega$ be admissible for $A$. Then $\Omega$ is admissible for $B$ and $m(B, \Omega)=m(A, \Omega)$.

Proof. First we show that $\Omega$ is admissible for $B$. Taking $z \in \partial \Omega$ we have the commutative diagram of vector-spaces and linear mappings

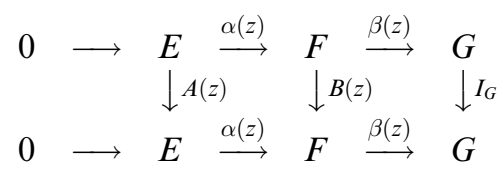

where $A(z)$ is invertible. From this it follows that $B(z)$ is invertible.

Now we turn to the main part of the proof. We abbreviate $\mathscr{H}(\Omega, E)$ to $\mathscr{H}(E)$, with corresponding abbreviations for the other spaces $F$ and $G$. We have an induced map $\bar{\alpha}$ from $\mathscr{H}(E) / A \mathscr{H}(E)$ to $\mathscr{H}(F) / B \mathscr{H}(F)$ given by

$$
\bar{\alpha}(f+A \mathscr{H}(E))=\alpha f+B \mathscr{H}(F)
$$

We shall show that $\bar{\alpha}$ is a Banach-space isomorphism. If $h \in \mathscr{H}(F)$ and $h(z) \in \operatorname{ran} \alpha(z)$ for each $z \in \bar{\Omega}$, then there exists $f \in \mathscr{H}(E)$ such that $\alpha f=h$. This last claim seems quite transparent, yet it needs proof, since we are not assuming that $\operatorname{ran} \alpha(z)$ is complemented in $F$. The proof is relegated to a lemma following the rest of the proof of this theorem.

To show that $\bar{\alpha}$ is injective: Suppose that $\bar{\alpha}(f+A \mathscr{H}(E))=0$. Then 
$\alpha f=B h$ for some $h \in \mathscr{H}(F)$. Hence $0=\beta \alpha f=\beta B h=\beta h$ so that $h(z) \in \operatorname{ker} \beta(z)=\operatorname{ran} \alpha(z)$ for each $z \in \Omega$. Hence, by the lemma, $h=\alpha g$ for some $g \in \mathscr{H}(E)$ and we find that $\alpha f=B h=B \alpha g=\alpha A g$; whence $f=A g$; that is $f+A \mathscr{H}(E)=0$.

To show that $\bar{\alpha}$ is surjective: Let $f \in \mathscr{H}(F)$. We have $f+B \mathscr{H}(F)=f-B f+B \mathscr{H}(F)$. Now $\beta(f-B f)=\beta f-\beta f=0$. Hence, by the lemma, $f-B f=\alpha g$ for some $g \in \mathscr{H}(E)$. That is, $f+B \mathscr{H}(F)=$ $\bar{\alpha}(g+A \mathscr{H}(E))$.

Lemma 5 (i) Let $X$ be a topological space. Let $E$ and $F$ be Banach spaces, $\alpha: X \rightarrow L(E, F)$ a continuous mapping such that $\alpha(z)$ is injective and has closed range for each $z \in X$. Let $h: X \rightarrow F$ be continuous and suppose that $h(z) \in \operatorname{ran} \alpha(z)$ for each $z \in X$. Then there exists a continuous mapping $f: X \rightarrow$ E such that $h=\alpha f$.

(ii) If, in addition, $X$ is an open subset of $\mathrm{C}$, and $\alpha$ and $h$ are analytic, then $f$ is analytic.

Proof. (i) Of course there exists a function $f$ such that $h=\alpha f$. The problem is to show that $f$ is continuous. Note that we are not assuming that the range of $\alpha(z)$ is complemented. For each $z$ the linear map $\alpha(z)$ has a continuous linear inverse defined on the closed, but variable, subspace ran $\alpha(z)$. Let us denote this inverse by $\gamma(z)$.

Fix $z_{0} \in X$. There exists a neighbourhood $N$ of $z_{0}$ such that for all $z \in N$ we have $\left\|\alpha(z)-\alpha\left(z_{0}\right)\right\|<\frac{1}{2}\left\|\gamma\left(z_{0}\right)\right\|^{-1}$. Let $u \in E$. For $z \in N$ we have $\|\alpha(z) u\| \geq\left\|\alpha\left(z_{0}\right) u\right\|-\frac{1}{2}\left\|\gamma\left(z_{0}\right)\right\|^{-1}\|u\| \geq\left\|\gamma\left(z_{0}\right)\right\|^{-1}\|u\|-\frac{1}{2}\left\|\gamma\left(z_{0}\right)\right\|^{-1}\|u\| \geq$ $\frac{1}{2}\left\|\gamma\left(z_{0}\right)\right\|^{-1}\|u\|$. Hence $\|\gamma(z)\| \leq 2\left\|\gamma\left(z_{0}\right)\right\|$ for all $z \in N$, whence $\|\gamma\|$ is locally bounded. We conclude that $f$ is locally bounded.

Now we have

$$
\left(\alpha(z)-\alpha\left(z_{0}\right)\right) f(z)+\alpha\left(z_{0}\right)\left(f(z)-f\left(z_{0}\right)\right)=h(z)-h\left(z_{0}\right)
$$

Since $h$ is continuous and $f$ is locally bounded we find, letting $z \rightarrow z_{0}$, that $\lim _{z \rightarrow z_{0}} f(z)-f\left(z_{0}\right)=0$, that is, $f$ is continuous.

(ii) We have

$$
\begin{gathered}
\left(\frac{\alpha(z)-\alpha\left(z_{0}\right)}{z-z_{0}}-\alpha^{\prime}\left(z_{0}\right)\right) f(z)+\alpha\left(z_{0}\right) \frac{f(z)-f\left(z_{0}\right)}{z-z_{0}}+\alpha^{\prime}\left(z_{0}\right) f(z) \\
=\frac{h(z)-h\left(z_{0}\right)}{z-z_{0}}
\end{gathered}
$$

whence, letting $z \rightarrow z_{0}$, and using the continuity of $f$, we obtain the differentiability, and hence analyticity, of $f$. 


\section{Some examples.}

In this section we compute $m(A, \Omega)$ in a number of special cases.

Theorem 9. Let $A: D \rightarrow \mathrm{C}$ be analytic. Then $m(A, \Omega)$ is the sum of the usual multiplicities of the zeros of $A$ in $\Omega$.

Proof. By axiom 5 we reduce this to the case when $A$ has one zero $z_{0}$ in $\Omega$. Let $A(z)=\phi(z)\left(z-z_{0}\right)^{r}$ where $\phi(z) \neq 0$ for all $z \in \Omega$. By the product theorem we have $m(A, \Omega)=\operatorname{rm}(B, \Omega)$ where $B(z)=z-z_{0}$. A trivial calculation gives $m(B, \Omega)=1$.

Let $T \in L(E, E)$. The operator-function $z \mapsto z I_{E}-T$ will be denoted by $L_{T}$. Now let $(A, \Omega)$ be an admissible pair. Then there exists a Banach space $F$ and an operator $T \in L(F, F)$ such that $A$ is s-equivalent to $L_{T}$ on some neighbourhood of $\bar{\Omega}$. We say that $L_{T}$ is a linearization of $A$ on $\Omega$. The GKLprocess described in [3] and based directly on [1] provides a linearization. In a succeeding section we shall describe another linearization.

In [3] the multiplicity of an admissible pair was defined by choosing a linearization $L_{T}$ for $A$ on $\Omega$ and then letting the multiplicity be the isomorphism class of the range of the spectral projection associated with $T$ and $\Omega$ by means of the operational calculus. The next result therefore implies that $m(A, \Omega)$ coincides with the multiplicity defined in [3].

THeOREM 10. $m\left(L_{T}, \Omega\right)$ is the isomorphism class of the range of the spectral projection associated with $T$ and $\Omega$.

Proof. Let $A=L_{T}$, let $E=E(A)$ and abbreviate $\mathscr{H}(\Omega, E)$ to $\mathscr{H}$. The results of section 2 indicate that the Banach space $\mathscr{H} / L_{T} \mathscr{H}$ is isomorphic to the subspace of $\mathscr{H}$ consisting of all functions of the form

$$
g_{1}(z)=\frac{1}{2 \pi i} \int_{\partial \Omega^{\prime}} A_{z}(\zeta) A(\zeta)^{-1} f(\zeta) d \zeta
$$

as $f$ ranges over $\mathscr{H}$, and that the mapping $f \mapsto g_{1}$ thus defined is a projection. Here we use the notation of section 2; in particular formula (1). Now $A_{z}=I_{E}$ and hence we have

$$
g_{1}(z)=\frac{1}{2 \pi i} \int_{\partial \Omega^{\prime}}\left(\zeta I_{E}-T\right)^{-1} f(\zeta) d \zeta
$$

Thus the function $g_{1}$ is a constant. But the result of projecting a constant $u$ is $(1 / 2 \pi i) \int_{\partial \Omega^{\prime}}\left(\zeta I_{E}-T\right)^{-1} u d \zeta=Q u$ where $Q$ is the spectral projection associated with $T$ and $\Omega$. Hence $\mathscr{H} / L_{T} \mathscr{H}$ is isomorphic to the range of $Q$.

It now follows that in the case where the values of $A$ are all Fredholm 
operators of index zero, $m(A, \Omega)$ is the sum of the multiplicities of the singular points of $A$ in $\Omega$. For the proof of this we refer the reader to [3].

\section{The canonical linearization.}

Let $(A, \Omega)$ be an admissible pair. Abbreviate $\mathscr{H}(\Omega, E)$ to $\mathscr{H}$ and $\mathscr{H}_{A}(\Omega, E)$ to $\mathscr{H}_{A}$. We let $\pi_{A}$ denote the canonical linear mapping from $\mathscr{H}$ to $\mathscr{H}_{A}$. On the space $\mathscr{H}_{A}$ we define a linear mapping $\omega_{A}$ by letting $\omega_{A} \pi_{A} f=\pi_{A} \omega f$, where $\omega$ is the linear map $\mathscr{H} \rightarrow \mathscr{H}$ given by $(\omega f)(w)=w f(w)$ for all $w \in \bar{\Omega}$. The function $z \mapsto z I_{\mathscr{H}_{A}}-\omega_{A}$ will be called the canonical linearization of the admissible pair $(A, \Omega)$.

There is a point here which may cause confusion. The canonical linearization is associated with $\Omega$, but its domain is all of C. It will appear later that the canonical linearization of $(A, \Omega)$ is a linearization of $A$ on a domain larger than $\Omega$.

Lemma 6. Let $A$ and $B$ be s-equivalent and let $\Omega$ be admissible for both. Then there exists an isomorphism $\gamma: \mathscr{H}_{A}(\Omega, E(A)) \rightarrow \mathscr{H}_{B}(\Omega, E(B))$ such that $\gamma \omega_{A}=\omega_{B} \gamma$.

Proof. It suffices to consider two cases. In the first case $B$ is a weak suspension of $A$. In the second case $B$ is equivalent to $A$.

Assume that $B$ is a weak suspension of $A$. Using the notation of the proof of theorem 8 we have an isomorphism $\bar{\alpha}: \mathscr{H}_{A}(\Omega, E(A)) \rightarrow \mathscr{H}_{B}(\Omega, E(B))$ induced by the linear map $\mathscr{H}(\Omega, E(A)) \rightarrow \mathscr{H}(\Omega, E(B))$ given by $f \mapsto \alpha f$. Since this map commutes with $\omega$ it is immediate that $\bar{\alpha} \omega_{A}=\omega_{B} \bar{\alpha}$; so we can let $\gamma=\bar{\alpha}$.

Assume next that $B$ is equivalent to $A$, say, $\phi A=B \psi$, where $\phi$ and $\psi$ are invertible. By the remark after theorem 7 there is an isomorphism from $\mathscr{H}_{A}(\Omega, E(A))$ to $\mathscr{H}_{B}(\Omega, E(B))$ induced by the linear map $\mathscr{H}(\Omega, E(A)) \rightarrow$ $\mathscr{H}(\Omega, E(B))$ given by $f \mapsto \phi f$. The proof is now completed as in the first case.

Theorem 11. Let $(A, \Omega)$ be an admissible pair. Then $A$ is s-equivalent to the canonical linearization of $(A, \Omega)$ on some neighbourhood of $\bar{\Omega}$.

Proof. Since $A$ is known to possess a linearization on a neighbourhood of $\bar{\Omega}$ (e.g. that given by the GKL-process), it suffices to show that, if $A$ is already linear, then it is s-equivalent to its canonical linearization. So let $A=L_{T}$ with $T \in L(E, E)$. By the results of the last section, $\pi_{A} f=\pi_{A} u$ where $u$ is the constant function $(1 / 2 \pi i) \int_{\partial \Omega^{\prime}}\left(\zeta I_{E}-T\right)^{-1} f(\zeta) d \zeta$, which, regarded as a vector, belongs to the range of the spectral projection $Q=(1 / 2 \pi i) \int_{\partial \Omega^{\prime}}\left(\zeta I_{E}-T\right)^{-1} d \zeta$. Hence 


$$
\omega_{A} \pi_{A} f=\omega_{A} \pi_{A} u=\pi_{A} \omega u=\pi_{A}\left(\frac{1}{2 \pi i} \int_{\partial \Omega^{\prime}}\left(\zeta I_{E}-T\right)^{-1} \zeta u d \zeta\right)=\pi_{A} T u
$$

Hence the operator $\omega_{A}$ on $\mathscr{H}_{A}$ is similar to the operator $T \mid$ ran $Q$. By spectral theory $L_{T}$ is equivalent to $L_{T \mid \operatorname{ran} Q} \oplus L_{T \mid \operatorname{ker} Q}$ on all of C. But $L_{T \mid \operatorname{ker} Q}$ is invertible on $\bar{\Omega}$. Hence $L_{T}$ is s-equivalent on a neighbourhood of $\bar{\Omega}$ to $L_{T \mid \operatorname{ran} Q}$, and hence to $L_{\omega_{A}}$. This concludes the proof.

It is clear that $z I_{E}-\omega$ is invertible for all $z \notin \bar{\Omega}$. The same, therefore, applies to $z I_{\mathscr{H}}-\omega_{A}$. It follows from theorem 11 that the spectrum of $\omega_{A}$ coincides with that part of the singular set of $A$ which lies within $\Omega$, a result which does not seem obvious. Another consequence of theorem 11 is that if $m(A, \Omega)=0$ then $\Sigma_{\Omega}(A)=\emptyset$. For if $m(A, \Omega)=0$ then the canonical linearization is defined on the trivial vector space $\{0\}$; hence the spectrum of $\omega_{A}$, which coincides with $\Sigma_{\Omega}(A)$, is empty.

The foregoing proof of theorem 11 is quite sufficient for most purposes. However we thought it of interest to give another proof, which is more constructive in that it exhibits an explicit s-equivalence between $A$ and its canonical linearization. The intervening spaces are probably of minimal size for a linearization. Compare this with the GKL-process (used in the proof of the last theorem) where it is always necessary to introduce a large space. The following result could therefore be useful if one was considering multiplicity theory for a restricted category of Banach spaces.

Theorem 12. Let $(A, \Omega)$ be an admissible pair and let $E=E(A)$. Then $A \oplus I_{\mathscr{H}_{A}(\Omega, E)}$ and $I_{E} \oplus L_{\omega_{A}}$ are equivalent on $\bar{\Omega}$ (that is, on an open neighbourhood of $\bar{\Omega})$.

The proof will occupy the rest of this section and will contain a number of lemmas. First we introduce some notation and make preliminary remarks. As usual $\mathscr{H}(\Omega, E)$ will be abbreviated to $\mathscr{H}$ and $\mathscr{H}_{A}(\Omega, E)$ to $\mathscr{H}_{A}$. We choose an admissible Cauchy-domain $\Omega^{\prime \prime}$ containing $\bar{\Omega}$ and such that $\Sigma_{\Omega^{\prime \prime}}(A)=\Sigma_{\Omega}(A)$. We shall in fact prove that $A \oplus I_{\mathscr{H}_{A}(\Omega, E)}$ and $I_{E} \oplus L_{\omega_{A}}$ are equivalent on $\Omega^{\prime \prime}$. For each $z \in \Omega^{\prime \prime}$ we define $B_{2}(z) \in L(E, E)$ by

$$
B_{2}(z)=\frac{1}{2 \pi i} \int_{\partial \Omega^{\prime \prime}} \frac{A(\zeta)^{-1}}{\zeta-z} d \zeta
$$

Then $B_{2}$ is an analytic $L(E, E)$-valued function on $\Omega^{\prime \prime}$.

For each $z \in \Omega^{\prime \prime}$ and $u \in E$ we define $K(z) \in L(E, \mathscr{H})$ by letting

$$
(K(z) u)(w)=A_{z}(w) u
$$

for all $w \in \bar{\Omega}$. Recall that $A_{z}(w)$ was defined in section 2 . Then $K$ is an ana- 
lytic $L(E, \mathscr{H})$-valued function on $\Omega^{\prime \prime}$. In fact $K$ is analytic on all of $D=D(A)$. To prove this it is enough to check that the mapping $z \mapsto K(z) u$ is an analytic $\mathscr{H}$-valued mapping for each $u \in E$ (see, for example, [8, page 206]). Fix $u \in E$ and let $F(z, w)=(K(z) u)(w)=A_{z}(w) u$. Then $K(z) u$ is the mapping $\bar{\Omega} \ni w \mapsto F(z, w)$, and we must show that the difference quotient $(F(z+h, w)-F(z, w)) / h$ attains its limit as $h \rightarrow 0$ uniformly with respect to $w \in \bar{\Omega}$. We have that $F$ is an analytic mapping from $D \times D$ to $E$. It follows that $D_{1} F$ is uniformly continuous on $\overline{\Omega^{\prime \prime}} \times \bar{\Omega}$. Hence the limit

$$
\lim _{h \rightarrow 0} \frac{F(z+h, w)-F(z, w)}{h}=D_{1} F(z, w)
$$

is attained uniformly with respect to $w \in \bar{\Omega}$. We conclude that the mapping $z \mapsto K(z) u$ is an analytic $\mathscr{H}$-valued mapping.

For each $f \in \mathscr{H}$ we define $U f \in E$ as follows. Choose a Cauchy-domain $\Omega^{\prime}$ containing $\Sigma_{\Omega}(A)$ but such that $\bar{\Omega}^{\prime} \subset \Omega$ and let

$$
U f=\frac{1}{2 \pi i} \int_{\partial \Omega^{\prime}} A(\zeta)^{-1} f(\zeta) d \zeta
$$

Clearly $U f$ does not depend on the choice of $\Omega^{\prime}$ subject to the stated conditions. Moreover $U$ is a continuous linear map from $\mathscr{H}$ to $E$.

If $f \in A \mathscr{H}$ then $U f=0$. Hence $U$ induces a continuous linear map $U_{A}: \mathscr{H}_{A} \rightarrow E$.

We denote by $J$ the embedding of $E$ into $\mathscr{H}$ which maps $u \in E$ to the constant function with value $u$.

We recall the linear map $G_{2}$ defined in section 2 . Here, though, we replace $\Omega$ by $\Omega^{\prime \prime}$, and then $G_{2}: \mathcal{O}\left(\Omega^{\prime \prime}, E\right) \rightarrow \mathcal{O}\left(\Omega^{\prime \prime}, E\right)$ is given by

$$
\left(G_{2} f\right)(z)=\frac{1}{2 \pi i} \int_{\partial \Omega^{\prime}} \frac{A(\zeta)^{-1} f(\zeta)}{\zeta-z} d \zeta
$$

where $\Omega^{\prime}$ is a Cauchy-domain such that $\bar{\Omega}^{\prime} \subset \Omega^{\prime \prime}$, and $\Omega^{\prime}$ contains $\Sigma_{\Omega}(A)$ and $z$. We recall the property of $G_{2}$ that if $f=A f_{1}$, with $f_{1} \in \mathcal{O}\left(\Omega^{\prime \prime}, E\right)$, then $G_{2} f=f_{1}$.

For each $z \in \Omega^{\prime \prime}$ we define $\chi(z) \in L(E \oplus \mathscr{H}, E \oplus \mathscr{H})$ by

$$
\chi(z)=\left(\begin{array}{cc}
I & U \\
0 & -I
\end{array}\right)\left(\begin{array}{cc}
I & 0 \\
-K(z) & I
\end{array}\right)=\left(\begin{array}{cc}
I-U K(z) & U \\
K(z) & -I
\end{array}\right)
$$

Clearly $\chi(z)$ is invertible and

$$
\chi(z)^{-1}=\left(\begin{array}{cc}
I & 0 \\
K(z) & I
\end{array}\right)\left(\begin{array}{cc}
I & U \\
0 & -I
\end{array}\right)=\left(\begin{array}{cc}
I & U \\
K(z) & K(z) U-I
\end{array}\right)
$$

Then $\chi$ is an analytic $L(E \oplus \mathscr{H}, E \oplus \mathscr{H})$-valued function on $\Omega^{\prime \prime}$. 
Since $U$ vanishes on $A \mathscr{H}$ we see that $\chi(z)$ and $\chi(z)^{-1}$ both map the subspace $\{0\} \oplus A \mathscr{H}$ to itself. It follows that $\chi(z)$ induces an isomorphism $\chi_{A}(z): E \oplus \mathscr{H}_{A} \rightarrow E \oplus \mathscr{H}_{A}$. Moreover $\quad \chi_{A}$ is an analytic $L\left(E \oplus \mathscr{H}_{A}\right.$, $E \oplus \mathscr{H}_{A}$ )-valued function on $\Omega^{\prime \prime}$.

For each $z \in \Omega^{\prime \prime}$ we define $\phi(z) \in L(E \oplus \mathscr{H}, E \oplus \mathscr{H})$ by

$$
\phi(z)=\left(\begin{array}{cc}
B_{2}(z) & U \\
J & \omega-z I
\end{array}\right)
$$

Then $\phi$ is an analytic $L(E \oplus \mathscr{H}, E \oplus \mathscr{H})$-valued function on $\Omega^{\prime \prime}$.

Since $U$ vanishes on $A \mathscr{H}$ we see that $\phi(z)$ maps $\{0\} \oplus A \mathscr{H}$ to itself. We conclude that $\phi(z)$ induces a continuous linear map $\phi_{A}(z): E \oplus \mathscr{H}_{A} \rightarrow$ $E \oplus \mathscr{H}_{A}$. Then $\phi_{A}$ is an analytic $L\left(E \oplus \mathscr{H}_{A}, E \oplus \mathscr{H}_{A}\right)$-valued function on $\Omega^{\prime \prime}$.

The proof of theorem 12 is completed in the following two steps:

Step 1. Proof that $\phi_{A}(z)$ is invertible for each $z \in \Omega^{\prime \prime}$.

Step 2. Proof that

$$
\phi_{A}(z)\left(\begin{array}{cc}
A(z) & 0 \\
0 & I
\end{array}\right)=\left(\begin{array}{cc}
I & 0 \\
0 & z I-\omega_{A}
\end{array}\right) \chi_{A}(z)
$$

for all $z \in \Omega^{\prime \prime}$. In short, $\phi_{A}\left(A \oplus I_{\mathscr{H}_{A}}\right)=\left(I_{E} \oplus L_{\omega_{A}}\right) \chi_{A}$.

To carry out step 1 we use the following lemma.

Lemma 7. Let $z \in \Omega^{\prime \prime}, u \in E$ and $f \in \mathcal{O}\left(\Omega^{\prime \prime}, E\right)$. Defineg $\in \mathcal{O}\left(\Omega^{\prime \prime}, E\right)$ by

$$
g(w)=u+(w-z) f(w)
$$

for all $w \in \Omega^{\prime \prime}$. Then

$$
G_{2}(g)(z)=B_{2}(z) u+U f
$$

Proof. Choose $\Omega^{\prime}$ so that $\bar{\Omega}^{\prime} \subset \Omega^{\prime \prime}, z \in \Omega^{\prime}$ and $\Sigma_{\Omega}(A) \subset \Omega^{\prime}$. Then

$$
G_{2}(g)(z)=\frac{1}{2 \pi i} \int_{\partial \Omega^{\prime}} \frac{A(\zeta)^{-1}}{\zeta-z}(u+(\zeta-z) f(\zeta)) d \zeta=B_{2}(z) u+U f
$$

Proof that $\phi_{A}(z)$ is injective

Every element of $E \oplus \mathscr{H}_{A}$ is represented by a pair $(u, f)$, where $u \in E$ and $f \in \mathcal{O}\left(\Omega^{\prime \prime}, E\right)$. Let the class of such a pair lie in the kernel of $\phi_{A}(z)$. Then $B_{2}(z) u+U f=0$, and there exists $f_{1} \in \mathcal{O}\left(\Omega^{\prime \prime}, E\right)$ such that $u+(w-z) f(w)=$ $A(w) f_{1}(w)$ for all $w \in \Omega^{\prime \prime}$. Using lemma 7 on the second equation we get $f_{1}(z)=B_{2}(z) u+U f$; and hence by the first equation $f_{1}(z)=0$. Hence there exists $f_{2} \in \mathcal{O}\left(\Omega^{\prime \prime}, E\right)$ such that $f_{1}(w)=(w-z) f_{2}(w)$ for all $w \in \Omega^{\prime \prime}$. By the 
second equation we get $u=0$ and $f=A f_{2}$. Hence the class of $(u, f)$ in $E \oplus \mathscr{H}_{A}$ is trivial.

Proof that $\phi_{A}(z)$ is surjective

Represent an element of $E \oplus \mathscr{H}_{A}$ by a pair $(v, g)$ where $v \in E$ and $g \in \mathcal{O}\left(\Omega^{\prime \prime}, E\right)$. Since we may replace $g$ by $g-A G_{2} g+A v$, and $G_{2}\left(g-A G_{2} g+A v\right)=v$, we may, without loss of generality, assume that $G_{2} g$ is the constant function $v$. We then let $u=g(z)$ and let $f \in \mathcal{O}\left(\Omega^{\prime \prime}, E\right)$ be determined by the equation $g(w)=g(z)+(w-z) f(w)$. By lemma 7 we then have $v=\left(G_{2} g\right)(z)=B_{2}(z) u+U f$. But then $\phi(z)$ maps $(u, f)$ to $(v, g)$.

Finally we carry out step 2 . We use the following lemma.

LemMa 8. For all $z \in \Omega^{\prime \prime}$

$$
B_{2}(z) A(z)+U K(z)=I
$$

Proof. Let $u \in E$. As we can consider $K(z) u$ as lying in $\mathcal{O}(D, E)$, we have

$$
\begin{aligned}
U K(z) u & =\frac{1}{2 \pi i} \int_{\partial \Omega^{\prime \prime}} A(\zeta)^{-1}(K(z) u)(\zeta) d \zeta \\
& =\frac{1}{2 \pi i} \int_{\partial \Omega^{\prime \prime}} A(\zeta)^{-1} A_{z}(\zeta) u d \zeta=\frac{1}{2 \pi i} \int_{\partial \Omega^{\prime \prime}} A(\zeta)^{-1} \frac{A(\zeta)-A(z)}{\zeta-z} u d \zeta \\
& =u-\left(\frac{1}{2 \pi i} \int_{\partial \Omega^{\prime \prime}} \frac{A(\zeta)^{-1}}{\zeta-z} d \zeta\right) u=u-B_{2}(z) A(z)
\end{aligned}
$$

Now we complete step 2 by calculating

$$
\begin{aligned}
& \phi(z)\left(\begin{array}{cc}
A(z) & 0 \\
0 & I
\end{array}\right)\left(\begin{array}{l}
u \\
f
\end{array}\right)-\left(\begin{array}{cc}
I & 0 \\
0 & z I-\omega
\end{array}\right) \chi(z)\left(\begin{array}{l}
u \\
f
\end{array}\right) \\
&=\left(\begin{array}{cc}
B_{2}(z) & U \\
J & \omega-z I
\end{array}\right)\left(\begin{array}{cc}
A(z) & 0 \\
0 & I
\end{array}\right)\left(\begin{array}{l}
u \\
f
\end{array}\right) \\
&-\left(\begin{array}{cc}
I & 0 \\
0 & z I-\omega
\end{array}\right)\left(\begin{array}{cc}
I-U K(z) & U \\
K(z) & -I
\end{array}\right)\left(\begin{array}{l}
u \\
f
\end{array}\right) \\
&=\left(\begin{array}{c}
B_{2}(z) A(z) u+U K(z) u-u \\
J A(z) u-(z I-\omega) K(z) u
\end{array}\right)=\left(\begin{array}{c}
0 \\
A u
\end{array}\right) \in\{0\} \oplus A \mathscr{H}
\end{aligned}
$$

where we have used lemma 8 at the very end, together with the formula $(J A(z)(w)-((z I-\omega) K(z) u)(w)=A(z) u+A(w) u-A(z) u=A(w) u$. This concludes the proof of theorem 12 . 


\section{Homotopy invariance.}

In this section we shall apply the considerations of section 2 , but allow $A$ to vary. Let $M_{\Omega}$ denote the space of all analytic mappings $A: \bar{\Omega} \rightarrow L(E, E)$, for which $A(z)$ is invertible for all $z \in \partial \Omega$. We consider $M_{\Omega}$ as a subset of the Banach space $\mathscr{H}(\Omega, L(E, E))$ with the induced topology. Denote the projection $S$ defined by equation (1) by $S_{A}$.

Lemma 9. $S_{A}$ depends continuously on $A \in M_{\Omega}$.

Proof. By the maximum principle $\|f(z)\|$ attains its maximum on the boundary $\partial \Omega$ for each $f \in \mathscr{H}(\Omega, E)$. Fix the Cauchy-domain $\Omega^{\prime}$ inside $\Omega$ in such a way that $|z-\zeta|$ is uniformly bounded below by a positive constant for all $z \in \partial \Omega$ and $\zeta \in \partial \Omega^{\prime}$. Consider now $A$ and $B$ in $M_{\Omega}$. For each $f \in \mathscr{H}(\Omega, E)$ there exists $z \in \partial \Omega$ such that

$$
\begin{aligned}
\left\|S_{A} f-S_{B} f\right\| & =\frac{1}{2 \pi}\left\|\int_{\partial \Omega^{\prime}}\left(\frac{A(\zeta)-A(z)}{\zeta-z} A(\zeta)^{-1}-\frac{B(\zeta)-B(z)}{\zeta-z} B(\zeta)^{-1}\right) f(\zeta) d \zeta\right\| \\
& =\frac{1}{2 \pi}\left\|\int_{\partial \Omega^{\prime}}(\zeta-z)^{-1}\left(B(z) B(\zeta)^{-1}-A(z) A(\zeta)^{-1}\right) f(\zeta) d \zeta\right\| \\
& \leq \frac{1}{2 \pi} \int_{\partial \Omega^{\prime}}|\zeta-z|^{-1}\left\|B(z) B(\zeta)^{-1}-A(z) A(\zeta)^{-1}\right\| d \zeta \cdot\|f\|
\end{aligned}
$$

whence we deduce

$$
\left\|S_{A}-S_{B}\right\| \leq C \sup _{\zeta \in \partial \Omega^{\prime}, z \in \partial \Omega}\left\|B(z) B(\zeta)^{-1}-A(z) A(\zeta)^{-1}\right\|
$$

where $C$ is independent of $A$ and $B$. It follows immediately that the mapping $A \mapsto S_{A}$ is continuous on $M_{\Omega}$.

Fix an initial $A$. By standard facts about projections on Banach spaces (see the lemma at the end of this section), there exists a neighbourhood $U$ of $A$ in $M_{\Omega}$ such that, for all $B \in U$, the restriction of $S_{A}$ to $\operatorname{ran} S_{B}$ is an isomorphism of the latter subspace onto $\operatorname{ran} S_{A}$. Hence the isomorphism class of $\operatorname{ran} S_{A}$ is a locally constant function on $M_{\Omega}$. Thus we obtain the homotopy invariance of $m(A, \Omega)$.

In fact we can say more. Abbreviate $\mathscr{H}(\Omega, E)$ to $\mathscr{H}$. Let $\mathscr{B}_{\Omega}$ denote the set of all pairs $(A, \theta)$ for $A \in M_{\Omega}$ and $\theta \in \mathscr{H} / A \mathscr{H}$. Then the mapping $(A, \theta) \mapsto A$ defines $\mathscr{B}_{\Omega}$ as a vector-bundle over $M_{\Omega}$.

As it is difficult to give a precise reference for the required material on projections, we include a proof here of somewhat more than is needed.

Lemma 10. Let $X$ be a Banach space and $P \in L(X, X)$ a continuous projection. Then there exists $\epsilon>0$ such that, if $Q \in L(X, X)$ is a projection and 
$\|P-Q\|<\epsilon$, then the restriction of $P$ to $\operatorname{ran} Q$ is an isomorphism of $\operatorname{ran} Q$ onto $\operatorname{ran} P$; and the restriction of $Q$ to $\operatorname{ran} P$ is an isomorphism of $\operatorname{ran} P$ onto $\operatorname{ran} Q$.

Proof. Let $Q$ be a projection and consider the operator $T=P Q+$ $(I-P)(I-Q)$. If $Q=P$ then $T=I$. Moreover $T-I=2 P Q-P-Q=$ $P(Q-P)+(P-Q) Q$. Hence $\|T-I\| \leq\|P-Q\|(\|P\|+\|Q\|)$. In the same way letting $T^{\prime}=Q P+(I-Q)(I-P)$ we have $\left\|T^{\prime}-I\right\| \leq\|P-Q\|$ $(\|P\|+\|Q\|)$. Recall that $P$ is fixed. Since the expression $\|P-Q\|$ $(\|P\|+\|Q\|)$ depends continuously on $Q$, there exists $\epsilon>0$ such that $\|P-Q\|(\|P\|+\|Q\|)<1$ whenever $Q$ is a projection which satisfies $\|P-Q\|<\epsilon$. For all such projections $Q$ the operators $T$ and $T^{\prime}$ are both invertible. Consider $T$. We have $T|\operatorname{ran} Q=P| \operatorname{ran} Q$ and $T \mid \operatorname{ker} Q=$ $(I-P) \mid \operatorname{ker} Q$. Hence $P(\operatorname{ran} Q) \oplus(I-P)(\operatorname{ker} Q)=T(\operatorname{ran} Q) \oplus T(\operatorname{ker} Q)=X$. However $P(\operatorname{ran} Q) \subset \operatorname{ran} P,(I-P)(\operatorname{ker} Q) \subset \operatorname{ker} P$, and $\operatorname{ran} P \oplus \operatorname{ker} P=X$. Hence the restriction of $P$ to $\operatorname{ran} Q$ is an isomorphism, and $P(\operatorname{ran} Q)=\operatorname{ran} P$. Applying a similar argument using $T^{\prime}$ we find that the restriction of $Q$ to $\operatorname{ran} P$ is an isomorphism of $\operatorname{ran} P$ onto $\operatorname{ran} Q$.

A corollary of this is the familiar fact that, if $P_{t}$ is a projection depending continuously on a variable $t$, then the isomorphism class of the range of $P_{t}$ is independent of $t$.

\section{Estimates of multiplicity.}

We can use ideas related to the canonical linearization to get an estimate of the size of the space $\mathscr{H}_{A}$, and hence the multiplicity.

THeORem 13. Let $A$ be a polynomial of degree $n$ with coefficients in $L(E, E)$. Let $\Omega$ be admissible for $A$. Then

$$
\mathscr{H}_{A}=\sum_{k=0}^{n-1} \omega_{A}^{k} \pi_{A}(E)
$$

Note that we regard $E$ as a subspace of $\mathscr{H}(\Omega, E)$ by identifying vectors with constant functions. The theorem generalizes the conclusion of section 6 , that in case $A$ is of the first degree in $z$, the space $\mathscr{H}_{A}$ may be computed by projecting constant functions alone. In the present case, $\mathscr{H}_{A}$ may be computed by projecting polynomials of degree less than $n$. Let us denote the space of all $E$-valued polynomials of degree less than or equal to $n$ by $\mathscr{P}_{n}(E)$. Clearly $\mathscr{P}_{n}(E)$ is a Banach space isomorphic to $E^{n+1}$. It is also in a natural way a subspace of $\mathscr{H}(\Omega, E)$. We shall as usual write $E$ for $\mathscr{P}_{0}(E)$. The conclusion of theorem 13 can then be written $\mathscr{H}_{A}=\pi_{A}\left(\mathscr{P}_{n-1}(E)\right)$. 
Proof. By the results of section 2 it is enough to show that the function

$$
g_{1}(z)=\frac{1}{2 \pi i} \int_{\partial \Omega^{\prime}} A_{z}(\zeta) A(\zeta)^{-1} f(\zeta) d \zeta
$$

is a polynomial of degree less than $n$ for every $f \in \mathscr{H}(\Omega, E)$. Recall that $A_{z}(\zeta)=(A(\zeta)-A(z)) /(\zeta-z)$. Hence, since $A(z)$ is a polynomial of degree $n$, the function $A_{z}(\zeta)$ is a polynomial in $z$ of degree less than $n$. The same is therefore true of $g_{1}(z)$.

We now give an estimate of the size of $m(A, \Omega)$ in the general case.

TheOREm 14. Let $A: D \rightarrow L(E, E)$ be analytic and let $\Omega$ be admissible for $A$. Then there exists $N$ such that $m(A, \Omega)$ is the isomorphism class of a complemented subspace of $E^{N}$. Moreover $\mathscr{H}_{A}=\pi_{A}\left(\mathscr{P}_{N-1}(E)\right)=\sum_{k=0}^{N-1} \omega_{A}^{k} \pi_{A}(E)$.

Proof. We first consider the case when $A$ is a rational function with poles outside $\Omega$. Write $A(z)=p(z)^{-1} B(z)$ where $B$ is an $L(E, E)$-valued polynomial, and $p$ is a $\mathrm{C}$-valued polynomial with all its zeros outside $\bar{\Omega}$. Since $p(z) \neq 0$ for all $z \in \Omega$ and $A=B p^{-1}$, we have that $A \mathscr{H}=B \mathscr{H}$ and so $\pi_{A}=\pi_{B}$. Let $N$ be the degree of $B$. We then have, using theorem 13: $\mathscr{H}_{A}=\pi_{A}(\mathscr{H})=\pi_{B}(\mathscr{H})=\pi_{B}\left(\mathscr{P}_{N-1}(E)\right)=\pi_{A}\left(\mathscr{P}_{N-1}(E)\right)$. This is the second conclusion of the theorem. Moreover from $\mathscr{H}_{A}=\pi_{B}(\mathscr{H})$ we see that $\mathscr{H}_{A}$ is isomorphic to $\operatorname{ran} S_{B}$. But the latter is a subspace of $\mathscr{P}_{N-1}(E)$ according to the proof of theorem 13. Hence $\operatorname{ran} S_{B}=S_{B}\left(\mathscr{P}_{N-1}(E)\right)$. Since $S_{B}$ is a continuous projection we conclude that $\operatorname{ran} S_{B}$ is a complemented subspace of $\mathscr{P}_{N-1}(E)$. Since the latter is isomorphic to $E^{N}$ we deduce that $m(A, \Omega)$ is the isomorphism class of a complemented subspace of $E^{N}$.

Now consider the case of analytic $A$. Without loss of generality we may assume that $\Omega$ is a Cauchy-domain. As shown in section $7, S_{B}$ depends continuously on $B$. Hence, by lemma 10, there exists $\epsilon>0$ such that, if $\|A(z)-B(z)\|<\epsilon$ for all $z \in \bar{\Omega}$ then the restriction of $S_{A}$ to $\operatorname{ran} S_{B}$ is an isomorphism of the latter onto $\operatorname{ran} S_{A}$. By Runge's theorem we can find a rational function $B$ that satisfies this condition. For such a $B$ we have that $m(A, \Omega)$ is the isomorphism class of $\operatorname{ran} S_{B}$, and the latter is isomorphic to a complemented subspace of $E^{N}$, for some $N$. Moreover $\mathscr{H}_{A}=\pi_{A}\left(\operatorname{ran} S_{B}\right)=$ $\pi_{A}\left(\mathscr{P}_{N-1}(E)\right)$ for some $N$. This concludes the proof.

Theorem 14 implies at a stroke a number of conclusions, some easily obtainable by other means, others by no means obvious. For example: if $E$ is finite-dimensional, then so is $m(A, \Omega)$; if $E$ is a Hilbert space, then $m(A, \Omega)$ is the class of some Hilbert space; if $E$ is reflexive, then so is $m(A, \Omega)$. 


\title{
REFERENCES
}

1. I. C. Gohberg, M. A. Kaashoek \& D. C. Lay, Equivalence, linearization, and decomposition of holomorphic operator functions, J. Funct. Anal. 28 (1978), 102-144.

2. M. A. Kaashoek, C. V. M. van der Mee \& L. Rodman, Analytic operator functions with compact spectrum. I. Spectral nodes, linearization and equivalence, Integral Equations Operator Theory (1981), 504-547.

3. Robert Magnus, On the multiplicity of an operator-valued analytic function, Math. Scand. 77 (1995), 108-118.

4. R. J. Magnus, A generalization of multiplicity and the problem of bifurcation, Proc. London Math. Soc. 32 (1976), 251-278.

5. A. S. Markus \& E. I. Sigal, The multiplicity of the characterisic number of an operator valued function, Mat. Issled. 5 vyp. 3 (17) (1970), 129-147.

6. A. Friedman \& M. Shinbrot, Non-linear eigenvalue problems, Acta Math. 121 (1968), 77-125.

7. A. E. Taylor, Spectral theory of closed distributive operators, Acta Math. 84 (1950), 189-224.

8. A. E. Taylor, Introduction to Functional Analysis, John Wiley \& Sons Inc. New York 1958.

\author{
SCIENCE INSTITUTE \\ UNIVERSITY OF ICELAND \\ DUNHAGA 3 \\ 107 REYKJAVIK \\ ICELAND
}

